Copyright (C 2014 IEEE. Personal use of this material is permitted. Permission from IEEE must be obtained for all other uses, in any current or future media, including reprinting/republishing this material for advertising or promotional purposes, creating new collective works, for resale or redistribution to servers or lists, or reuse of any copyrighted component of this work in other works. 


\title{
Intrinsic Geometries and Properties of Piezo-MEMS Power Harvesters with Tip Mass Offset using New Electromechanical Finite Element Vibration Analysis
}

\author{
Mikail F. Lumentut, Member, IEEE, and Ian M. Howard
}

\begin{abstract}
Autonomous self-powered wireless sensor devices are inevitable future technology that will potentially become ubiquitous in many sectors such as industry, intelligent infrastructure and biomedical devices. This has spurred a great attention from researchers to develop self-sustained power harvesting devices. For this paper, we present a new numerical technique for modelling the MEMS power harvesters using parametric design optimisation and physical properties for various piezoelectric materials. This technique enables the prediction of optimal power harvesting responses that can be used to identify the performance of piezoelectric materials and particular piezoelectric geometry where this technique can alleviate tedious analytical methods for analysing parametric design optimisation and can assist for analysing piezo-MEMS system response before conducting the micro-fabrication process.
\end{abstract}

\section{INTRODUCTION}

Harvesting electrical energy from the surrounding vibration environment can enable sensor devices to be completely self-sustaining. Such a situation requires technical challenges for designing robust power harvesters based on the geometry, physical properties, micro-fabrication, power optimisation, power management electronic circuits and sensor systems. The majority of piezoelectric power harvesting research has been focused on the meso-scale device for predicting power output using various analytical techniques such as electromechanical lumped parameter models and electrical equivalent system [1], [2], analytical approach using weak form techniques [3], [4] and closed form techniques [5]-[7]. The typical cantilever piezoelectric unimorph and bimorph beams with tip mass have been widely used for generating high power harvesting, since they allow significant electrical energy to be produced due to high elemental strain from the transverse bending motion. This technique has also drawn attention from researchers for the design of MEMS power harvesters using microfabrication processes which mainly involve sputter deposition, photolithography, and etch processes. Most notably element layers of the device consist of active

M.F. Lumentut and I.M. Howard are with the Laboratory for Dynamic Systems of Smart Structure and Vibration, Department of Mechanical Engineering, Curtin University, Australia.

(emails:m.lumentut@exchange.curtin.edu.au/mikail_lumentut@yahoo.com andi.howard@curtin.edu.au) piezoelectric material covered by electrode layers and passive silicon wafers with silicon proof mass [8]-[13].

The vast majority of MEMS power harvesting devices utilise piezoelectric materials made from lead zirconate titanate (PZT) and aluminum nitrate (AIN). The benefit of using PZT is that it can extract high power output due to its physical properties, having large piezoelectric coupling coefficient and dielectric permittivity. On the other hand, AIN is lead free material, having low piezoelectric coefficient and dielectric permittivity, but gives higher power density with moderate voltage output where the fabrication method provides for the use of the easy sputter deposition process [12], [13]. So far, there are a number of published papers concentrating on micro-fabrication of power harvesters. However, only a few research efforts have investigated MEMS power harvesters using analytical studies and fabrication design [11], [13].

In this paper, the development of piezo-MEMS power harvesters using parametric geometry design and the physical properties will be explored using the new electromechanical finite element vibration method. At this stage, there are no other researchers developing this new numerical technique for modelling the piezo-MEMS power harvester. The key equations of constitutive finite element analysis are revealed and simplified, outlining the technical process for analysing optimal power harvesting frequency responses using different material properties and geometrical parameters of piezoelectric materials. The proposed numerical technique has proved that tedious analytical solutions can be avoided especially for analysing current parametric design optimisation and the prediction of the power harvesting performance can easily be simulated before conducting the fabrication process of the device.

\section{EleCtromechanicAl Finite ElEMENT PIEZOELECTRIC ENERGY HARVESTER}

In the microfabrication system considered here, the piezo-MEMS under base excitation and variable load resistance are covered with the electrode layers that can be connected with the two wires for generating one single voltage output as shown in Fig. 1. In this case, for numerical modelling, the global finite element equations of the system in Fig. 2 are based on the mechanical discretised element and the electrical discretised element where this is called the electromechanical discretisation. 


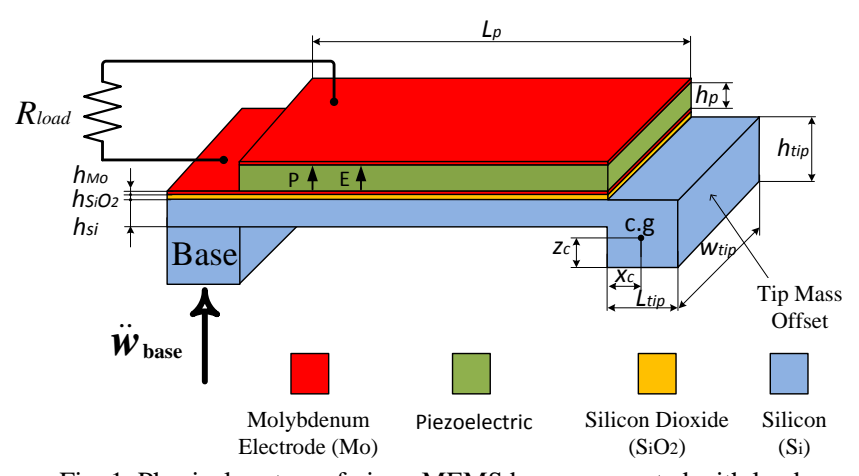

Fig. 1. Physical system of piezo-MEMS beam connected with load resistance under base motion

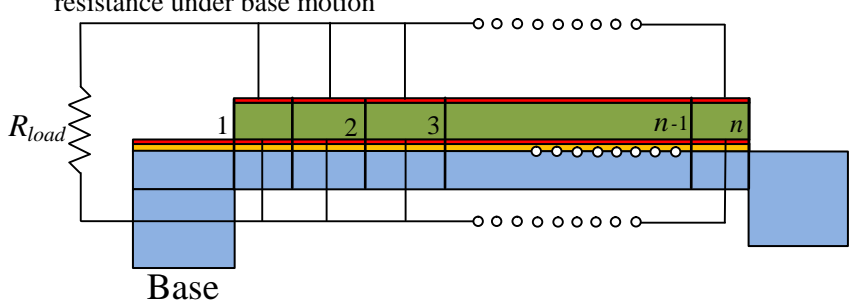

Fig. 2. Electromechanical discretisation of piezo-MEMS beam

\section{A. Electromechanical coupled finite element formulation}

The linear coupled field piezoelectric constitutive equations based on the 3-1 mode of piezoelectric constant operation and 3-3 effect of piezoelectric permittivity can be formulated as,

$$
\begin{gathered}
\boldsymbol{T}_{1}^{(2)}=\overline{\mathbf{c}}_{11}^{(2)} \boldsymbol{S}_{1}^{(2)}-\mathbf{e}_{31} \boldsymbol{E}_{3}, \\
\boldsymbol{D}_{3}=e_{31} \boldsymbol{S}_{1}^{(2)}+\varepsilon_{33}^{S} \boldsymbol{E}_{3} .
\end{gathered}
$$

The linear-elastic constitutive relation for the substructure can also be formulated as,

$$
\boldsymbol{T}_{1}^{(1)}=\overline{\mathbf{c}}_{11}^{(1)} \boldsymbol{S}_{1}^{(1)}
$$

where the parameters $\boldsymbol{T}, \boldsymbol{S}, \boldsymbol{E}$ and $\boldsymbol{D}$ represent stress, strain, electric field, and electric displacement, respectively. Moreover, coefficients $c, e$, and $\varepsilon$ indicate elastic constant, piezoelectric coefficient, and permittivity at constant strain, respectively. The superscript 1 and 2 represent substructure and piezoelectric layers, respectively.

The solution form of the mechanical transverse beam element can be formulated using the first-order Hermite interpolation function to give,

$$
\boldsymbol{w}(x, t)=\boldsymbol{\Phi}^{e}(x) \boldsymbol{u}^{e}(t) .
$$

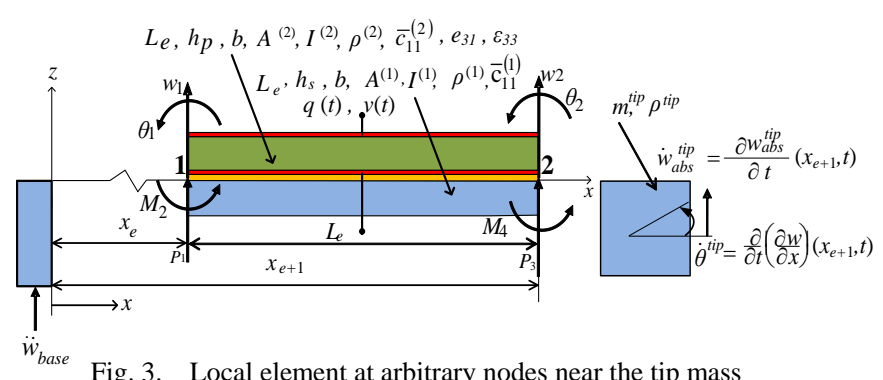

Parameters of the shape function $\boldsymbol{\Phi}$ and the elemental displacement vector $\boldsymbol{u}$ for each node based on Fig. 3 can be formulated as, where,

$$
\begin{gathered}
\boldsymbol{\Phi}^{e}(x)=\left[\begin{array}{llll}
\Phi_{1}(x) & \Phi_{2}(x) & \Phi_{3}(x) & \Phi_{4}(x)
\end{array}\right], \\
\boldsymbol{u}^{e}(t)=\left[\begin{array}{llll}
u_{1} & u_{2} & u_{3} & u_{4}
\end{array}\right]^{\mathrm{T}},
\end{gathered}
$$

$$
\begin{gathered}
\Phi_{1}(x)=1-3\left(\frac{x-x_{e}}{L_{e}}\right)^{2}+2\left(\frac{x-x_{e}}{L_{e}}\right)^{3}, \Phi_{2}(x)=\left(x-x_{e}\right)\left(1-\frac{x-x_{e}}{L_{e}}\right)^{2}, \\
\Phi_{3}(x)=3\left(\frac{x-x_{e}}{L_{e}}\right)^{2}-2\left(\frac{x-x_{e}}{L_{e}}\right)^{3}, \Phi_{4}(x)=\left(x-x_{e}\right)\left[\left(\frac{x-x_{e}}{L_{e}}\right)^{2}-\frac{x-x_{e}}{L_{e}}\right], \\
L_{e}=x_{e+1}-x_{e}, \quad u_{1}(t)=w_{1}(t), \quad u_{2}(t)=\theta_{1}(t), \\
u_{3}(t)=w_{2}(t), u_{4}(t)=\theta_{2}(t) .
\end{gathered}
$$

The strain-displacement relationship in terms of the vector displacement can be expressed as,

$$
\boldsymbol{S}(x, t)=-\mathrm{z} \boldsymbol{\Psi}^{e}(x) \boldsymbol{u}^{e}(t),
$$

where the differential form of the shape function $\Psi$ of the strain displacement relationship can be formulated as,

$$
\boldsymbol{\Psi}^{e}(x)=\frac{\mathrm{d}^{2} \boldsymbol{\Phi}^{e}(x)}{\mathrm{d} x^{2}}=\left[\begin{array}{llll}
\Psi_{1}(x) & \Psi_{2}(x) & \Psi_{3}(x) & \Psi_{4}(x)
\end{array}\right] .
$$

The solution from the discretised electric field $\boldsymbol{E}$ can be assumed to be linear along the thickness of the piezoelectric material for inducing electrical potential $\phi$ over the piezoelectric element. The electric field can be formulated as,

$$
\boldsymbol{E}_{3}=-\nabla \boldsymbol{\phi}^{e}(z, t)=-\boldsymbol{\Omega}^{e}(z) \boldsymbol{\nu}^{e}(t) .
$$

Note that electrical shape function was given as $\boldsymbol{\Omega}^{e}(z)=1 / h_{p}$. The stress fields in the partial differential shape function forms can be expressed by substituting Eq. (6) and Eq. (8) into Eq. (1a) and Eq. (2) to give,

$$
\begin{gathered}
\boldsymbol{T}_{1}^{(2)}=-\mathbf{z} \overline{\boldsymbol{c}}_{11}^{(2)} \boldsymbol{\Psi}^{e}(\mathbf{x}) \boldsymbol{u}^{e}(\mathbf{t})+\mathbf{e}_{31} \boldsymbol{\Omega}(z) \boldsymbol{v}^{e}(t), \\
\boldsymbol{T}_{1}^{(1)}=-\mathbf{z} \bar{c}_{11}^{(1)} \boldsymbol{\Psi}^{e}(x) \boldsymbol{u}^{e}(t) .
\end{gathered}
$$

The electric displacement vector of the piezoelectric component can be formulated by substituting Eqs. (6)-(8) into Eq. (1b) to give,

$$
\boldsymbol{D}_{3}=z e_{31} \Psi^{e}(x) \boldsymbol{u}^{e}(t)-\boldsymbol{\varepsilon}_{33}^{S} \Omega^{e}(z) \boldsymbol{v}^{e}(t) .
$$

\section{B. Lagrangian electromechanical finite element equations}

Lagrange equation for deriving the electromechanical discretised finite element dynamic equations of the piezoMEMS power harvester can be formulated as,

$$
\sum_{s=1}\left[\frac{\mathrm{d}}{\mathrm{d} t} \frac{\partial \Pi}{\partial \boldsymbol{\eta}_{\mathrm{s}}}-\frac{\partial \Pi}{\partial \boldsymbol{\eta}_{\mathrm{s}}}-\boldsymbol{F}_{s}\right]=0,
$$

where $\Pi=K E-P E+W E, \quad \eta_{s} \in\{\dot{\boldsymbol{u}}(t), \boldsymbol{u}(t), \boldsymbol{v}(t)\}$, and $\boldsymbol{F}_{\mathbf{s}} \in\left\{\ddot{\boldsymbol{w}}_{\text {base }}(t), \boldsymbol{q}(t)\right\}$ or $\left\{\sum_{s=1} F_{\mathrm{s}}\right\}=\left\{\frac{\partial W F}{\partial u^{e}(t)}+\frac{\partial W F}{\partial v^{e}(t)}\right\}$.

The kinetic energy can be formulated from the mass densities of the piezoelectric element and tip mass as,

$$
K E=\frac{1}{2} \int_{x_{e} A^{(1)}}^{x_{e+1}} \rho^{(1)} \dot{\boldsymbol{w}}(x, t)^{T} \dot{\boldsymbol{w}}(x, t) \mathrm{d} A^{(1)} \mathrm{d} x
$$




$$
\begin{aligned}
& +\frac{1}{2} \int_{x_{\mathrm{e} A} A^{(2)}}^{x_{e+1}} \rho^{(2)} \dot{\boldsymbol{w}}(x, t)^{T} \dot{\boldsymbol{w}}(x, t) \mathrm{d} A^{(2)} \mathrm{d} x+I_{0}^{t i p} x_{c} \dot{\boldsymbol{w}}\left(x_{e+1}, t\right)^{T} \dot{\boldsymbol{\theta}}\left(x_{e+1}, t\right) \\
& +\frac{1}{2} I_{0}^{t i p} \dot{\boldsymbol{w}}\left(x_{e+1}, t\right)^{T} \dot{\boldsymbol{w}}\left(x_{e+1}, t\right)+\frac{1}{2} I_{2}^{t i p} \dot{\boldsymbol{\theta}}\left(x_{e+1}, t\right)^{T} \dot{\boldsymbol{\theta}}\left(x_{e+1}, t\right) .
\end{aligned}
$$

Note that the effect of the rotary inertia of the tip mass $I_{2}^{t i p}$ including its offset parameters $I_{0}^{\text {tip }} x_{c}$ are taken into account where most previous major published works have ignored this case. Parameter $I_{0}^{\text {tip }}$ is the zeroth mass moment of inertia of the tip mass.

The potential energy for the piezoelectric element can be formulated as,

$$
P E=\frac{1}{2} \int_{x_{e}}^{x_{e}+1} \int_{A^{(1)}} S_{1}^{(1)^{T}} \boldsymbol{T}_{1}^{(1)} \mathrm{dA}^{(1)} \mathrm{d} x+\frac{1}{2} \int_{x_{e}}^{x_{e+1}} \int_{A^{(2)}} \boldsymbol{S}_{1}^{(2)^{T}} \boldsymbol{T}_{1}^{(2)} \mathrm{dA}^{(2)} \mathrm{d} x .
$$

The electrical energy term for the piezoelectric element can be formulated as,

$$
W E=\frac{1}{2} \int_{x_{e} A^{(2)}}^{x_{e+1}} \int_{3} \boldsymbol{E}^{T} \boldsymbol{D}_{3} \mathrm{~d} A^{(2)} \mathrm{d} x
$$

The non-conservative work on the system due to the input base excitation and electrical charge output can be stated as,

$$
\begin{aligned}
& W F=-\int_{x_{e} A^{(1)}}^{x_{e+1}} \rho^{(1)} \boldsymbol{w}(x, t)^{T} \mathrm{~d} A^{(1)} \mathrm{d} x \ddot{\boldsymbol{w}}_{\text {base }}(t) \\
& -\int_{x_{e} A^{(2)}}^{x_{e+1}} \rho^{(2)} \boldsymbol{w}(x, t)^{T} \mathrm{~d} A^{(2)} \mathrm{d} x \ddot{\boldsymbol{w}}_{\text {base }}(t)-\boldsymbol{I}_{0}^{t i p} x_{c} \boldsymbol{\theta}\left(x_{e+1}, t\right)^{T} \ddot{\boldsymbol{w}}_{\text {base }}(t) \\
& \quad-\boldsymbol{I}_{0}^{t i p} \boldsymbol{w}\left(x_{e+1}, t\right)^{T} \ddot{\boldsymbol{w}}_{\text {base }}(t)+\boldsymbol{q}^{(e)}(t) \boldsymbol{v}^{(e)}(t) .
\end{aligned}
$$

The expression given from Eq. (11) can be further solved by substituting Eqs. (3)-(10) to give the local element matrices of electromechanical dynamic equations by including Rayleigh damping as,

$$
\left[\begin{array}{cc}
\boldsymbol{M}^{e} & 0 \\
0 & 0
\end{array}\right]\left\{\begin{array}{l}
\ddot{\boldsymbol{u}}^{e}(t) \\
\ddot{\boldsymbol{v}}^{e}(t)
\end{array}\right\}+\left[\begin{array}{cc}
\boldsymbol{C}^{e} & 0 \\
\boldsymbol{P}_{\theta}^{e^{T}} & \boldsymbol{P}_{D}^{e}
\end{array}\right]\left\{\begin{array}{c}
\dot{\boldsymbol{u}}^{e}(t) \\
\dot{\boldsymbol{v}}^{e}(t)
\end{array}\right\}+\left[\begin{array}{cc}
\boldsymbol{K}^{e} & \boldsymbol{P}_{\theta}^{e} \\
0 & 0
\end{array}\right]\left\{\begin{array}{c}
\boldsymbol{u}^{e}(t) \\
\boldsymbol{v}^{e}(t)
\end{array}\right\}=\left\{\begin{array}{c}
\boldsymbol{F}^{e} \\
\boldsymbol{i}^{e}
\end{array}\right\},(16
$$

where

$$
\begin{gathered}
\boldsymbol{M}^{e}=\sum_{i=1}\left(\int_{x_{e}}^{x_{e+1}} \int_{A^{(i)}} \rho^{(i)} \boldsymbol{\Phi}^{e}(x)^{T} \boldsymbol{\Phi}^{e}(x) \mathrm{d} A^{(i)} \mathrm{d} x\right)+I_{0}^{t i p} \boldsymbol{\Phi}^{e}\left(x_{e+1}\right)^{T} \boldsymbol{\Phi}^{e}\left(x_{e+1}\right) \\
+2 I_{0}^{t i p} x_{c} \boldsymbol{\Phi}^{e}\left(x_{e+1}\right)^{T} \frac{\mathrm{d} \boldsymbol{\Phi}^{e}\left(x_{e+1}\right)}{\mathrm{d} x}+I_{2}^{t i p} \frac{\mathrm{d} \boldsymbol{\Phi}^{e}\left(x_{e+1}\right)^{T}}{\mathrm{~d} x} \frac{\mathrm{d} \boldsymbol{\Phi}^{e}\left(x_{e+1}\right)}{\mathrm{d} x}, \\
\boldsymbol{K}^{e}=\sum_{i=1}\left(\int_{x_{e} A^{(i)}}^{x_{e+1}} z^{2} \bar{c}_{11}^{(i)} \boldsymbol{\Psi}^{e}(x)^{T} \boldsymbol{\Psi}^{e}(x) \mathrm{d} A^{(i)} \mathrm{d} x\right), \boldsymbol{C}^{e}=\alpha \boldsymbol{M}^{e}+\beta \boldsymbol{K}^{e}, \\
\boldsymbol{P}_{\theta}^{e}=-\sum_{p=1}\left(\int_{x_{e} A^{(p)}}^{x_{e+1}} z e_{31}^{(p)} \boldsymbol{\Psi}^{e}(x)^{T} \boldsymbol{\Omega}^{e}(z) \mathrm{d} A^{(p)} \mathrm{d} x\right),
\end{gathered}
$$

$$
\begin{gathered}
\boldsymbol{P}_{\theta}^{e^{T}}=-\sum_{p=1}\left(\int_{x_{e} A^{(p)}}^{x_{e+1}} z e_{3 l}^{(p)} \boldsymbol{\Omega}^{e}(z)^{T} \boldsymbol{\Psi}^{e}(x) \mathrm{d} A^{(p)} \mathrm{d} x\right), \\
\boldsymbol{P}_{D}^{e}=-\sum_{p=1}\left(\int_{x_{e} A^{(p)}}^{x_{e+1}} \varepsilon_{33}^{(p)} \boldsymbol{\Omega}^{e}(z)^{T} \boldsymbol{\Omega}^{e}(z) \mathrm{d} A^{(p)} \mathrm{d} x\right), \boldsymbol{F}^{e}=-\boldsymbol{Q}^{e} \ddot{\boldsymbol{w}}_{\text {base }}(t), \\
\boldsymbol{Q}^{(e)}=\sum_{i=1}^{x_{e+1}} \int_{x_{e} A^{(i)}} \rho^{(i)} \boldsymbol{\Phi}^{e}(x)^{T} \mathrm{~d} A^{(i)} \mathrm{d} x+I_{0}^{t i p} x_{c} \frac{\mathrm{d} \boldsymbol{\Phi}^{e}\left(x_{e+1}\right)^{T}}{\mathrm{~d} x}+I_{0}^{t i p} \boldsymbol{\Phi}^{e}\left(x_{e+1}\right)^{T} .
\end{gathered}
$$

\section{Global matrices of electromechanical dynamic equation}

The global matrix forms can be formulated using the generalised dynamic equations to give,

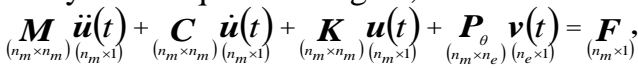

$$
\begin{aligned}
& \underset{\left(n_{e} \times n_{m}\right)}{\boldsymbol{P}_{\left(n_{m} \times 1\right)}^{\boldsymbol{T}}} \underset{\boldsymbol{u}}{\dot{u}(t)}+\underset{\left(n_{e} \times n_{e}\right)}{\boldsymbol{P}_{\boldsymbol{b}}} \underset{\left(n_{e} \times 1\right)}{\dot{\boldsymbol{v}}(t)}=\underset{\left(n_{e} \times 1\right)}{\boldsymbol{i}(t)},
\end{aligned}
$$

where $\boldsymbol{M}$ is global mass matrix, $\boldsymbol{K}$ is global stiffness matrix and $\boldsymbol{C}$ is Rayleigh damping. Other parameters $\boldsymbol{P}_{\theta}, \boldsymbol{P}_{D}$, and $\boldsymbol{F}$ indicate global electromechanical coupling matrices, global capacitance matrices, global mechanical forces, respectively. Moreover, variable $\boldsymbol{i}$ is global current output, $\boldsymbol{u}$ is global mechanical coordinate, and $v$ is global voltage output.

\section{Solution techniques using the orthonormalised global scalar forms}

The solution form of Eq. (17) can be formulated in terms of the normalised modal vector and time-dependent displacement generalised coordinate as,

$$
\boldsymbol{u}(t)=\boldsymbol{\varphi}_{1} \boldsymbol{a}_{1}(t)+\boldsymbol{\varphi}_{2} \boldsymbol{a}_{2}(t)+\ldots+\boldsymbol{\varphi}_{m-1} \boldsymbol{a}_{m-1}(t)+\boldsymbol{\varphi}_{m} \boldsymbol{a}_{m}(t)=\boldsymbol{\varphi} \boldsymbol{a}(t),
$$

where the normalised modal matrix $\varphi$ can be formulated as,

$$
\boldsymbol{\varphi}=\boldsymbol{U}\left(\boldsymbol{U}^{\boldsymbol{T}} \boldsymbol{M U}\right)^{-\frac{1}{2}},
$$

where $\boldsymbol{U}$ represents eigenvector. Equation (17) can be further formulated by substituting Eq. (18) and premultiplying the result by $\varphi^{T}$. The result of which can simply be formulated as,

$$
\begin{aligned}
\boldsymbol{\varphi}^{T} \boldsymbol{M} \varphi \ddot{\mathrm{a}}(\mathbf{t})+\boldsymbol{\varphi}^{T} \boldsymbol{C} \boldsymbol{\varphi} \dot{\mathrm{a}}(\mathbf{t})+\boldsymbol{\varphi}^{T} \boldsymbol{K} \boldsymbol{\varphi} \mathrm{a}(\mathbf{t})+\boldsymbol{\varphi}^{T} \boldsymbol{P}_{\boldsymbol{\theta}} \boldsymbol{v}(\mathbf{t})=\boldsymbol{\varphi}^{T} \boldsymbol{F}, \\
\boldsymbol{P}_{\theta}^{T} \dot{\boldsymbol{a}}(t)+\boldsymbol{P}_{\boldsymbol{D}} \dot{\boldsymbol{v}}(t)=\boldsymbol{i}(t),
\end{aligned}
$$

where orthonormalised parameters from Eq. (20) can be stated as,

$$
\begin{gathered}
\boldsymbol{\varphi}^{T} \boldsymbol{M} \boldsymbol{\varphi}=\boldsymbol{I}, \quad \boldsymbol{\varphi}^{T} \boldsymbol{K} \boldsymbol{\varphi}=\omega^{2}, \\
\boldsymbol{\varphi}^{T} \boldsymbol{C} \boldsymbol{\varphi}=\alpha\left(\boldsymbol{\varphi}^{T} \boldsymbol{M} \boldsymbol{\varphi}\right)+\beta\left(\varphi^{T} \boldsymbol{K} \boldsymbol{\varphi}\right)=\alpha \boldsymbol{I}+\beta \boldsymbol{\omega}^{2}=2 \zeta \omega, \\
\hat{\boldsymbol{P}}_{\theta}=\boldsymbol{\varphi}^{T} \boldsymbol{P}_{\theta}, \quad \hat{\boldsymbol{P}}_{\theta}^{T}=\boldsymbol{P}_{\theta}^{T} \boldsymbol{\varphi}, \quad \hat{\boldsymbol{Q}}=\boldsymbol{\varphi}^{T} \boldsymbol{Q} .
\end{gathered}
$$

It should be noted that equations (21a) and (21b) represent the orthornormality property of mechanical dynamic equations that show diagonal matrices. At this case, equation (20) can be simplified as

$$
\begin{gathered}
\ddot{a}(t)+2 \zeta \omega \dot{a}(t)+\omega^{2} a(t)+\hat{\boldsymbol{P}}_{\theta} v(t)=-\hat{Q}_{\ddot{w}_{\text {base }}}(t), \\
\hat{\boldsymbol{P}}_{\theta}^{T} \dot{\boldsymbol{a}}(t)+\boldsymbol{P}_{D} \dot{v}(t)=i(t) .
\end{gathered}
$$


Global scalar form of the electromechanical dynamic equations can be further formulated using Eq. (22) in order to obtain the series form of the multimode FRFs. In this case, the first discretised electromechanical piezoelectric dynamic equation can be formulated in terms of the multi degree of freedom (multimode) system $r=1,2,3 \ldots N D O F$ and the number of normalised piezoelectric elements $s=1,2,3 \ldots N E L P$ as,

$$
\begin{gathered}
\ddot{a}_{r}(t)+2 \zeta_{r} \omega_{r} \dot{a}_{r}(t)+\omega_{r}^{2} a_{r}(t)+\sum_{s=1}^{N E L P} \hat{P}_{r s} v_{s}(t)=-\hat{Q}_{r} \ddot{w}_{\text {base }}(t), \\
r=1,2, \ldots, N D O F .
\end{gathered}
$$

The second form of the discretised electromechanical piezoelectric dynamic equation can be formulated as,

$$
\sum_{r=1}^{N D O F} \hat{P}_{s r} \dot{a}_{r}(t)+P_{D s} \dot{v}_{s}(t)=i_{P s}(t), s=1,2,3, \ldots, N E L P .
$$

The internal parallel connection in terms of the electrical discretised elements using Kirchhoff's voltage law (KVL) and Kirchhoff's current law (KCL) must be formulated in the scalar form as,

$$
\begin{gathered}
v_{1}(t)=v_{2}(t)=\ldots . .=v_{s}(t)=v(t), \\
i_{p 1}(t)+i_{p 2}(t)+\ldots+i_{p s}(t)=i_{p}(t) .
\end{gathered}
$$

Voltage output related to the external load resistance can be formulated as,

$$
v(t)=i_{p}(t) R_{\text {load }} .
$$

In this stage, after applying mathematical derivations using Eqs. (23)-(26), the power harvesting multimode FRFs series related to the input transverse acceleration can be formulated as,

$$
\frac{P_{p}(j \omega)}{\left(-\omega^{2} w_{\text {base }} e^{j \omega t}\right)^{2}}=\left[\frac{\frac{1}{\sqrt{R_{\text {load }}}} \sum_{r=1}^{N D O F} \frac{\hat{Q}_{r} j \omega \sum_{s=1}^{N E L P} \hat{P}_{s r}}{\omega_{r}{ }^{2}-\omega^{2}+j 2 \zeta_{r} \omega_{r} \omega}}{\sum_{s=1}^{N E L P} j \omega P_{D s}-\frac{1}{R_{\text {load }}}-\sum_{r=1}^{N D O F} \frac{j \omega \sum_{s=1}^{N E L P} \hat{P}_{s r} \sum_{s=1}^{N E L P} \hat{P}_{r s}}{\omega_{r}{ }^{2}-\omega^{2}+j 2 \zeta_{r} \omega_{r} \omega}}\right]^{2}
$$

To formulate the optimal multimode FRF of power harvesting, the reduced optimal load resistance can be formulated by differentiating Eq. (27) with respect to load resistance and setting the formula to be zero to give,

$$
R_{\text {lood }}^{\text {opt }}=\frac{\sqrt{X(\omega)^{2}+Y(\omega)^{2}}}{X(\omega)^{2}+Y(\omega)^{2}},
$$

where $X(\omega)=\sum_{s=1}^{N E L P} \omega P_{D s}-\sum_{r=1}^{N D O F} \frac{\omega \sum_{s=1}^{N E L P} \hat{P}_{s r} \sum_{s=1}^{N E L P} \hat{P}_{r s}\left(\omega_{r}{ }^{2}-\omega^{2}\right)}{\left(\omega_{r}{ }^{2}-\omega^{2}\right)^{2}+\left(2 \zeta_{r} \omega_{r} \omega\right)^{2}}$,

$$
Y(\omega)=\sum_{r=1}^{N D O F} \frac{\omega \sum_{s=1}^{N E L P} \hat{P}_{s r} \sum_{s=1}^{N E L P} \hat{P}_{r s}\left(2 \zeta_{r} \omega_{r} \omega\right)}{\left(\omega_{r}^{2}-\omega^{2}\right)^{2}+\left(2 \zeta_{r} \omega_{r} \omega\right)^{2}} .
$$

It should be noted that the optimal power harvesting can also be obtained by substituting Eq. (28) into Eq. (27).

\section{RESUlt AND Discussion}

Piezo-MEMS device with different material properties and geometrical parameters are modelled using the proposed numerical technique. The selected piezoelectric properties are listed in table 1 . The input base transverse acceleration onto the device was chosen to be $7 \mathrm{~m} / \mathrm{s}^{2}$. In Fig. 4, the device length $L$ and width $b$ with piezoelectric thickness $h_{p}$ and substructure (silicon) thickness $h_{s}$ were set to $5 \mathrm{~mm}, 2$ $\mathrm{mm}, 1 \mu \mathrm{m}$ and $9 \mu \mathrm{m}$, respectively. The tip mass configurations were calculated according to material property made from silicon with density of $2330 \mathrm{~kg} / \mathrm{m}^{3}$ and Young's modulus of $160 \mathrm{GPa}$ where the dimensions of tip mass with length $l_{t i p}$, thickness $h_{t i p}$ and width $w_{t i p}$ were set to $4 \mathrm{~mm}, 0.4 \mathrm{~mm}$ and $2 \mathrm{~mm}$, respectively.

The optimal power harvesting FRFs of the piezo-MEMS configurations using Eqs. (28) and (29) are performed for identifying the optimal frequency bandwidths based on the physical properties of piezoelectric materials and for analysing the vibration characteristics. In Fig. 4, the device response using piezoelectric material made from PZN-PT shows the characteristic power harvesting FRF under variable load resistance. It also shows that the optimal power harvesting FRF whose amplitudes coincides with the load resistances of $1.2 \mathrm{k} \Omega$ and $1 \mathrm{M} \Omega$ gives two maximum values with the same result. Further investigation of the optimal power harvesting FRFs using different material properties

\begin{tabular}{|c|c|c|c|c|c|}
\hline $\begin{array}{l}\text { Reference/ } \\
\text { Company }\end{array}$ & $\begin{array}{l}\text { Piezo- } \\
\text { electric } \\
\text { material }\end{array}$ & $\begin{array}{l}\text { Young's } \\
\text { modulus } \\
\overline{\mathrm{c}}_{11}(\mathrm{GPa})\end{array}$ & $\begin{array}{l}\text { Piezo- } \\
\text { electric } \\
\text { coefficient } \\
\mathrm{d}_{31}(\mathrm{pm} / \mathrm{V})\end{array}$ & $\begin{array}{c}\text { Relative } \\
\text { dielectric } \\
\text { constant } \\
\varepsilon_{33}^{\mathrm{T}} / \varepsilon_{\mathrm{o}}\end{array}$ & $\begin{array}{l}\text { Density } \\
\left(\mathrm{kg} / \mathrm{m}^{3}\right)\end{array}$ \\
\hline \multirow{2}{*}{$\begin{array}{l}\text { Andosca, et } \\
\text { al [13] }\end{array}$} & AIN & 340 & -2 & 9 & 3260 \\
\hline & PVDF & 3 & 20 & 12 & 1780 \\
\hline \multirow{2}{*}{$\begin{array}{l}\text { Piezo } \\
\text { Systems, } \\
\text { Inc }\end{array}$} & $\begin{array}{l}\text { PSI- } \\
5 \mathrm{~A} 4 \mathrm{E}\end{array}$ & 66 & -190 & 1800 & 7800 \\
\hline & $\begin{array}{l}\text { PSI- } \\
5 \mathrm{H} 4 \mathrm{E}\end{array}$ & 62 & -320 & 3800 & 7800 \\
\hline \multirow{3}{*}{$\begin{array}{l}\text { APC Inter- } \\
\text { national, } \\
\text { Ltd }\end{array}$} & $\begin{array}{l}\text { PMN- } \\
32 \% \mathrm{PT}\end{array}$ & 24.77 & -930 & 4600 & 8200 \\
\hline & $\begin{array}{l}\text { APC } \\
840\end{array}$ & 80 & -125 & 1275 & 7600 \\
\hline & $\begin{array}{l}\text { APC } \\
855\end{array}$ & 59 & -276 & 3300 & 7600 \\
\hline Microfine & $\begin{array}{l}\text { PZN- } \\
\text { PT }\end{array}$ & 25 & -1200 & 6500 & 8000 \\
\hline \multirow{2}{*}{$\begin{array}{l}\text { DeL Piezo } \\
\text { Specialities }\end{array}$} & DL-40 & 100 & -48 & 350 & 7700 \\
\hline & DL-53 & 61 & -275 & 3350 & 7600 \\
\hline
\end{tabular}
can be seen in Fig. 5a, where most of the system responses show two peaks of equal amplitude with different size of frequency bandwidths except the system responses from AIN and PVDF properties. In Fig. 5b, the frequency bandwidths for each MEMS power harvesting device can be used to identify the performance of the electromechanical system.

Table 1. Piezoelectric material properties. 


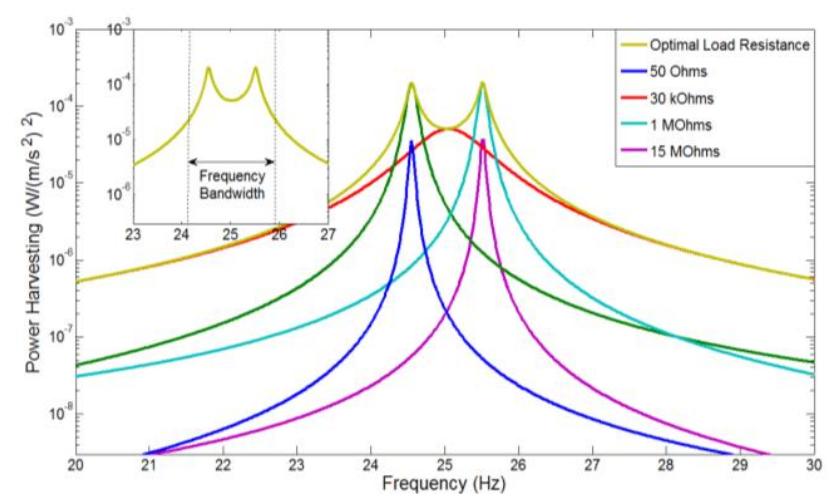

Fig. 4. Power harvesting FRF from PZN-PT.
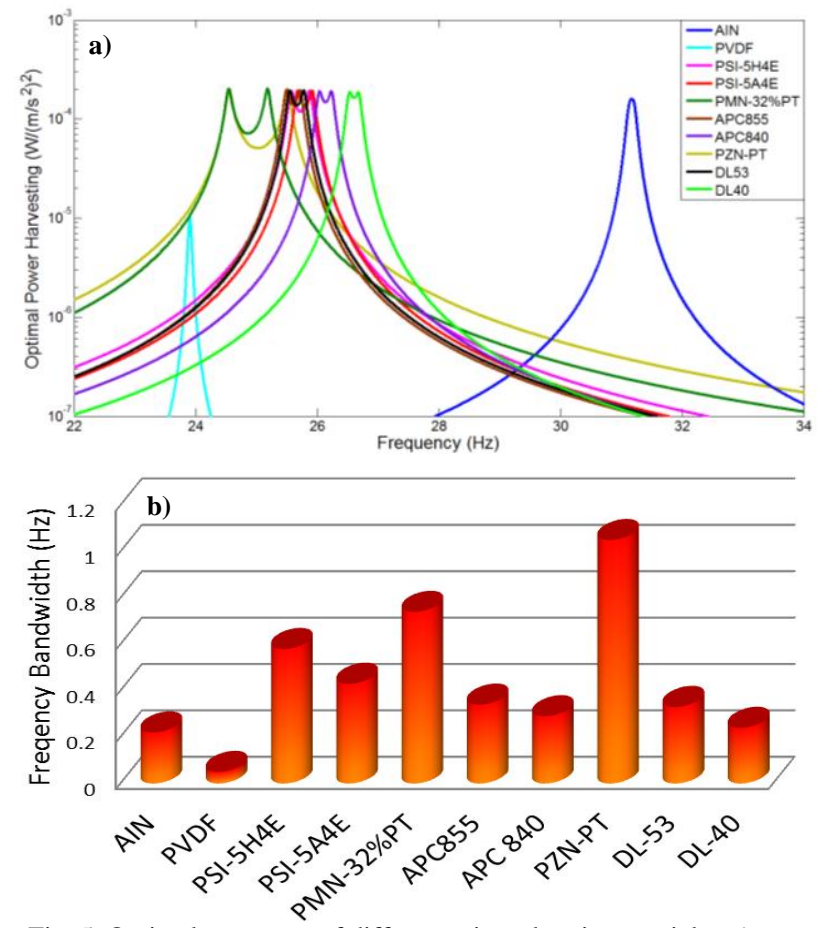

Fig. 5. Optimal responses of different piezoelectric materials : a) power harvesting FRFs and b) optimal frequency bandwidth.

The optimal frequency bandwidth obtained from the optimal power harvesting FRF depends on the optimal load resistance which is directly related to piezoelectric coupling coefficient and permittivity. If the Piezo-MEMS harvester has a wider optimal frequency bandwidth, the resonance frequency of the device can shift along its working frequency band using the resistive shunt circuit. That gives benefit when the frequency of the vibration environment is slightly away from the system response of the device. As shown in Figs. 5a-b, device response with PZN-PT gives the strongest electromechanical system whereas PVDF shows the weakest electromechanical system. In this case, the effect of higher piezoelectric coupling coefficient with higher permittivity provides stronger electromechanical system that encompasses a wider optimal frequency bandwidth. a)
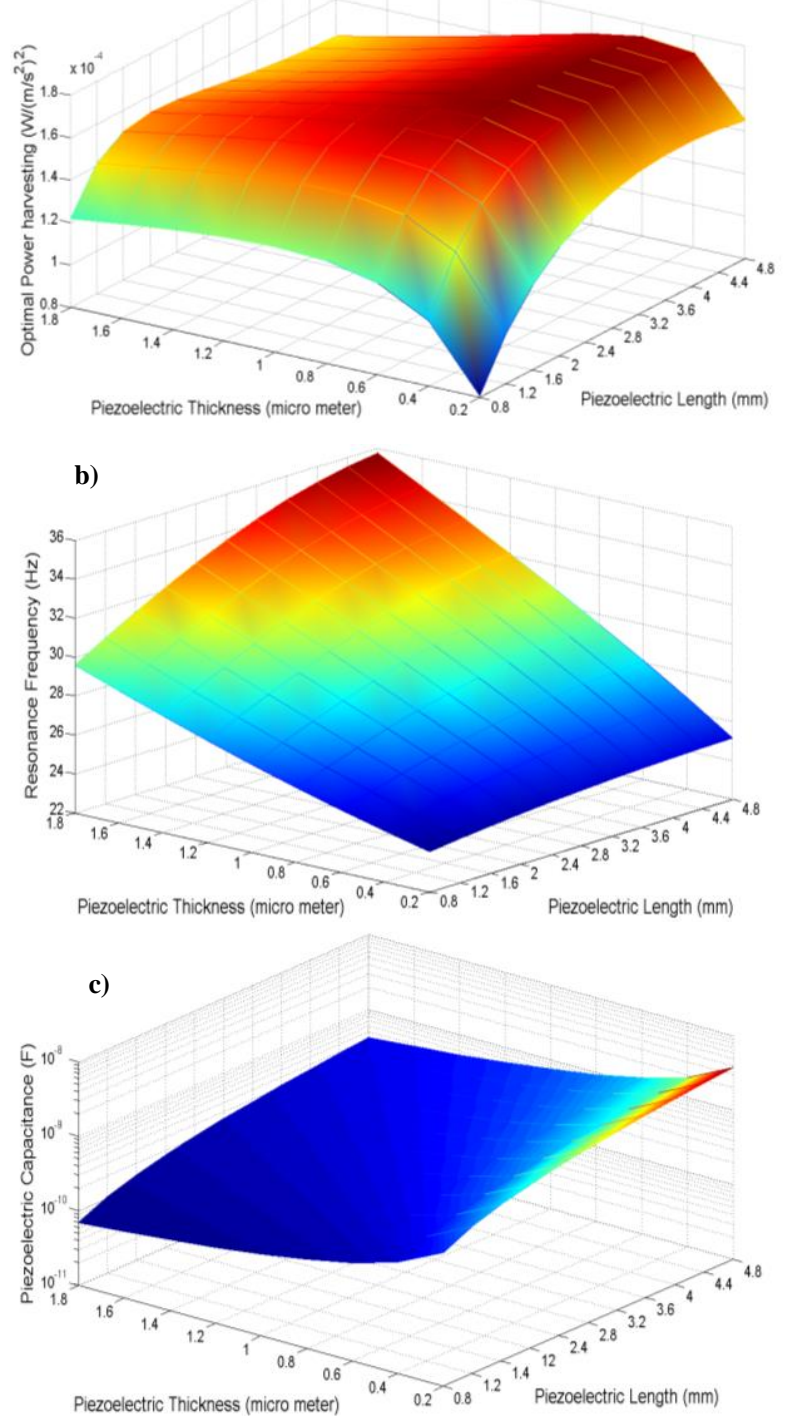

Fig. 6. Optimal parametric design of AIN piezoelectric: a) power harvesting FRFs, b) resonance frequency and c) piezoelectric capacitance.

Electromechanical vibration-based optimal power harvesting can also be further explored using the parametric design analyses based on the variations of piezoelectric length $\left(l_{p}\right)$, thickness $\left(h_{p}\right)$ and capacitance $\left(P_{D}\right)$ and tip mass length $\left(l_{t i p}\right)$ where the geometry of the silicon substrate as given earlier remains to be constant. For this case, AIN piezoelectric material was chosen. Although it shows very low optimal frequency bandwidth compared with other piezoelectric materials, it is a lead-free material with low dielectric permittivity and piezoelectric coupling constant that gives high power density, voltage and easy preparation of sputter deposition process for MEMS level [13]. In Figs. 6a-c, prediction of the optimal power harvesting using the parametric design of the piezoelectric length and thickness can be used to identify a particular location of the maximum level of the power output at certain resonance frequency. It shows that only a portion of the piezoelectric segment 
lengths between $3.2 \mathrm{~mm}$ and $4 \mathrm{~mm}$ with the thicknesses between $0.4 \mu \mathrm{m}$ and $0.8 \mu \mathrm{m}$ produces higher power output
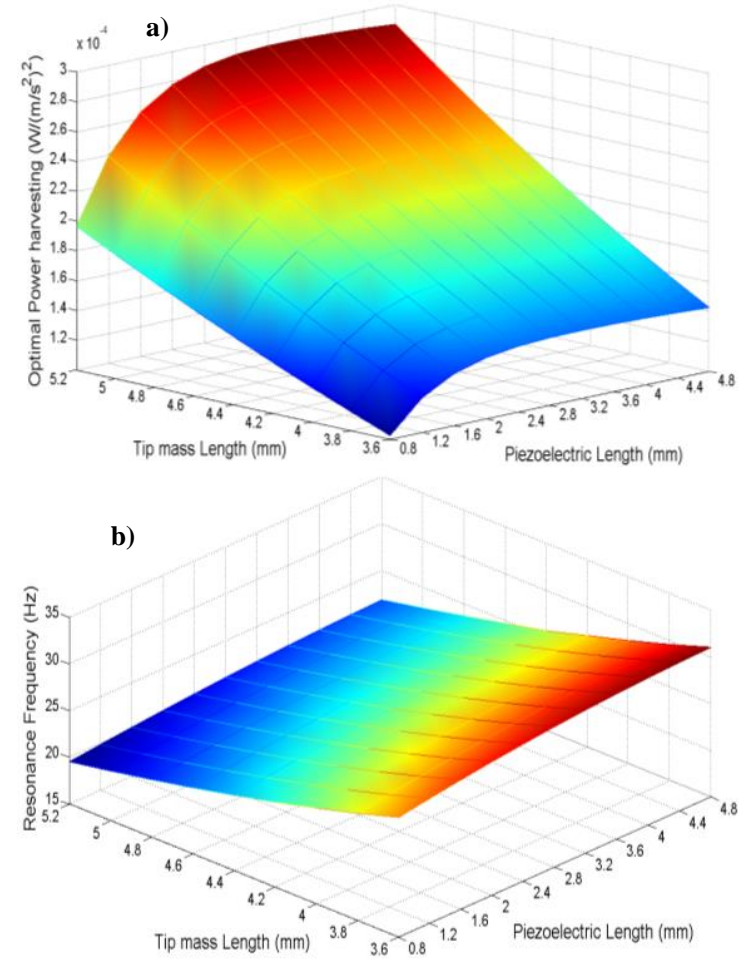

Fig. 7. Optimal parametric design of AIN piezoelectric and tip mass: a) optimal power harvesting amplitude and b) resonance frequency

with the frequency ranges from $24 \mathrm{~Hz}$ to $29 \mathrm{~Hz}$ and higher internal capacitance. Note that the piezoelectric segment length is measured from the base to the end of the beam. Other important results can also be seen in Figs. 7a-b, where the system responses are based on the variances of the tip mass length and piezoelectric length. The results show that higher power outputs with lower resonance frequencies can be obtained when volumes of the tip mass increase and the piezoelectric segment lengths are identified in the particular dimensions away from the middle point. Overall, the investigation of parametric MEMS power harvester device with variable tip mass and piezoelectric geometries can be used to identify the maximum power output with low resonance frequency.

\section{CONCLUSION}

This paper has presented parametric properties and design of the Piezo-MEMS power harvesters with the tip mass offset using novel numerical techniques of electromechanical finite element vibration analysis for formulating orthonormalised scalar forms of electromechanical dynamic equations. Such techniques can alleviate tedious solution from the analytical method, especially for designing complex geometry systems. The results show that piezoelectric properties can affect the optimal frequency bandwidth that can be used to tune the working frequency band of the device using the shunt circuit techniques. If the optimal frequency bandwidth gives larger value, the electromechanical system of the device will give a strong effect. Instead of making comparison with other piezoelectric materials using the optimal frequency bandwidth, the AIN piezoelectric material shows promising response for generating high power amplitudes, even though the piezoelectric coupling constant and electric permittivity show very low values. The challenge is to find the maximum resonance frequency of the device based on the best-fit amplitude from the vibration environment. The parametric optimal design of the piezo-MEMS can be an important technique for predicting maximum power harvesting before conducting micro-fabrication processes.

\section{REFERENCES}

[1] S. Roundy and P. K. Wright, "A piezoelectric vibration based generator for wireless electronics”, Smart Mater. Struct., vol. 18, pp. 1131-1142, 2004.

[2] J. Liang and W.-H. Liao, "Impedance modeling and analysis for Piezoelec-tric energy harvesting systems" IEEE/ASME Trans. Mechatronics, vol. 17, no. 6, pp. 1145-1157, Dec., 2012.

[3] M. Kim, M. Hoegen, J. Dugundji and B. L. Wardle "Modeling and experi-mental verification of proof mass effects on vibration energy harvester per-formance" Smart. Mater. Struct., vol. 19, 2010, art. no. 045023.

[4] M.F. Lumentut and I.M. Howard, "Analytical and experimental comparisons of electromechanical vibration response of a piezoelectric bimorph beam for power harvesting,"Mech. Syst. Signal Process, vol. 36, no. 1, pp. 66-86, 2013.

[5] A.M. Wickenheiser, "Eigensolution of piezoelectric energy harvesters with geometric discontinuities: Analytical modelling and validation," $J$. Intel. Mat. Syst. Struct. vol. 24, pp. 729-744, 2013.

[6] M.F. Lumentut and I.M. Howard, "Electromechanical piezoelectric power harvester frequency response modelling using closed-form boundary value methods," IEEE/ASME Trans. on Mech., vol. 19, no 1, pp. $32-44,2014$.

[7] M.F. Lumentut, L.A. Francis and I.M. Howard, "Analytical Techniques for Broadband Multielectromechanical Piezoelectric Bimorph Beams with Multifrequency Power Harvesting", IEEE Transactions on Ultrasonics, Ferroelectrics and Frequency Control, vol. 59, no. 11, pp. 2555 - 2568, 2012.

[8] S. Dongna, J.-H. Park, J. Ajitsaria, S.-Y. Choe, H.C. Wikle and D.-J. Kim, "The design, fabrication and evaluation of a MEMS PZT cantilever with an integrated Si proof mass for vibration energy harvesting," J.Micromech. Microeng. vol. 18, 2008, art.no. 055017.

[9] H.B. Fang, J.Q. Liu, Z.Y. Xu, L. Dong, L. Wang, D. Chen, B.C. Cai And Y. Liu, "Fabrication and performance of MEMS-based piezoelectric power generator for vibration energy harvester," Microelectron. J. vol. 37, pp. 1280-1284, 2006.

[10] D. Isarakorn, D. Briand, P. Janphuang, A. Sambri, S. Gariglio, J.-M. Triscone, F. Guy, J. WReiner, C.H.Ahn and N.F. de Rooij, "The Realization and performance of vibration energy harvesting MEMS device based on an epitaxial piezoelectric thin film," Smart Mater. Struct. vol.20 (2011), art. no. 025015 ,

[11] L.M. Miller, E. Halvorsen, T. Dong and P.K. Wright, "Modeling and experimental verification of low-frequency MEMS energy harvesting from ambient vibrations," J. Micromech. Microeng. vol. 21, 2011, art.no. 045029.

[12] R. Elfrink, T.M. Kamel, M. Goedbloed, S. Matova, D. Hohlfeld, V.Y.Andel and V.R. Schaijk, "Vibration energy harvesting with Aluminum nitride-based piezoelectric devices," J. Micromech. Microeng. 19, 2009, art no. 094005.

[13] R. Andosca, T.G. McDonald, V. Genova, S. Rosenberg, J. Keating, C. Benedixen and J. Wu, "Experimental and theoretical studies on MEMS piezoelectric vibrational energy harvesters with mass loading," Sens. Actuators A, vol. pp. 178 76-87, 2012. 